\title{
Chromosomal Abnormalities in a Pineocytoma
}

M. Josefa Bello

Juan A. Rey

Jose M. de Campos

M. Elena Kusak

Tumors of the pineal region represent about $0.4-1 \%$ of intracranial neoplasms, including those tumors of germ cell origin (primarily germinomas and teratomas, which represent about $80 \%$ of pineal malignancies), and those tumors of the pineal parenchyma (pineocytoma and pineoblastoma). Pineocytoma is composed of relatively mature cells of the pineal parenchyma, whereas pineoblastoma represents a more primitive form resembling medulloblastoma. The rarity of these tumors would account for the scarcity of cytogenetic studies performed on pineocytoma-pineoblastoma; only four cases have been previously reported. Griffin et al. analyzed two pineoblastomas occurring following hereditary retinoblastoma. The first sample displayed abnormalities of chromosome 1: del(1)(p31p21) and der $\mathrm{t}(1 ; ?)(\mathrm{p} 36 ; ?)$, whereas a $1 \mathrm{q}+$ and a Dq + chromosome characterized the second sample. Sreekantaiah et al.found a del(11)(q13.1q13.5) as the sole deviation in one pineoblastoma, which also displayed a cell including a del(1)(p31p36) in addition to the $11 \mathrm{q}-$. Finally, Rainho et el. analyzed a pineocytoma characterized by numerical alterations (losses) involving chromosomes X, 5, 8, 11, 14, and 22, and structural rearrangements of chromosomes 1,3,12, and 22, as follows: inv(1)(p31q44); del(3)(q11); del(12)(q22); del(22)(q11.2).

As a part of a comprehensive analysis of the cytogenetic characteristics of nervous system tumors, we have performed chromosome studies on a sample derived from a pineocytoma, arising in a 42 -year-old male patient. A total of 67 metaphases were analyzed by a direct method ( 25 cells) or after 7 days of in vitro growth (42 cells), utilizing the methodology previously described in detail. Heterogeneous chromosomal complements, which ranged from 41 and 47 chromosomes, with a pseudodiploid modal number, were found. Although poor-quality chromosome preparations were obtained, clonal numerical deviations were observed (in at least seven cells) involving chromosomes $\mathrm{Y}, 10,18,21$, and 22 . Structural rearrangements were identified as follows: $\operatorname{der}(2) t(2 ; 12)(q 33-36 ; q 13)$ (23 cells);

del(6)(q13q16) (12 cells); del(11)(q23) (18 cells); add(12)(q24) (11 cells); mar (small metacentric) (19 cells). Our findings are partially in agreement with the cytogenetic data previously obtained on pineocytomas-pineoblastomas; no abnormalities of chromosome 1 were observed in our case, but we confirm the involvement of $11 \mathrm{q}$, although in a region (q23-qter) slightly more distal than that deleted in the case studied by Sreekantaiah et al. Losses of chromosome 22 and a deletion of the distal $12 q$ region were observed as clonal in the case reported here, as occurs in the tumor reported by Rainho et al. Although the cytogenetic data based on five samples do not allow firm conclusions to be drawn, an 
involvement of Ip, as well as deletions of monosomy for chromosome 11, deletions of the distal 12q, and monosomy or deletion of chromosome 22, seem to be the chromosomal abnormalities recurrently found (in at least two samples) in this type of neoplasm. Of these alterations, only those affecting chromosome 22 (determined as losses at the cytogenetic level and as loss of heterozygosity in molecular studies) have been consistently found in association with the development of other histologic subtypes of brain tumors such as meningiomas, neurinomas, and malignant gliomas. Whether or not genes located on chromosomes 1, 11, 12, and 22 play a role in tumorigenesis of pineocytoma/pineoblastoma awaits additional cytogenetic data on a larger series of this type of tumors. These studies will doubtless contribute to a more precise identification of the chromosomal regions non-randomly involved, providing clues about the genomic domains where genes participating in the development of these neoplasms may be located. 